\title{
WT1 Negative
}

National Cancer Institute

\section{Source}

National Cancer Institute. WT1 Negative. NCI Thesaurus. Code C153555.

Indicates that expression of WT1 has not been detected in a sample. 